\title{
Development of a Large Pneumatocele in a Patient Recovering from COVID-19 Pneumonitis
}

\author{
Frances Hampson ${ }^{1}$, Waleed Salih ${ }^{2}$, Jennifer Helm²,3 \\ ${ }^{1}$ Department of Radiology, Salford Royal NHS Foundation Trust, Salford, UK \\ ${ }^{2}$ Department of Respiratory Medicine, Salford Royal NHS Foundation Trust, Salford, UK \\ ${ }^{3}$ School of Medicine, University of Manchester, Manchester, UK
}

Received: 10/05/2021

Accepted: $12 / 05 / 2021$

Published: 28/06/2021

How to cite this article: Hampson F, Salih W, Helm J. Development of a large pneumatocele in a patient recovering from COVID-19 pneumonitis. EJCRIM 2021;8: doi:10.12890/2021_002605.

Conflicts of Interests: The authors declare there are no competing interests.

This article is licensed under a Commons Attribution Non-Commercial 4.0 License

\section{ABSTRACT}

A 39-year-old man presented with severe COVID-19 pneumonitis requiring hospital admission. He represented three days following discharge with sudden onset breathlessness and chest pain. Initial imaging suggested the presence of a left pneumothorax. Following further clinical decline a plan was made to insert a CT guided chest drain. However, imaging in the prone position for the procedure unexpectedly revealed a large left lower lobe pneumatocele with only a very small pneumothorax. Events and appearances suggest that this is a rare case of delayed COVID-19 pneumonitis-related pneumatocele formation. We will discuss the clinical significance of this entity.

\section{LEARNING POINTS}

- Pneumatocele formation should be considered in patients presenting with new respiratory symptoms after completing therapy for COVID-19 pneumonitis.

- Performing CT examinations with patients in different positions may be required to help exclude the possibility of pneumatocele formation when a loculated pneumothorax is suspected on the supine CT images.

\section{KEYWORDS}

COVID-19, CT, pneumothorax, pneumatocele

\section{CASE DESCRIPTION}

A 39-year-old previously healthy man presented with a five day history of diarrhoea, a dry cough, shortness of breath and myalgia. On initial assessment he had a temperature of $38^{\circ} \mathrm{C}$, a respiratory rate of 34 and an elevated CRP. His chest radiograph demonstrated bilateral lower zone and peripheral predominant airspace typical for infection with COVID-19.

He was admitted and given oxygen via a face mask. The suspected diagnosis of COVID-19 was confirmed by reverse transcription polymerase chain reaction. He soon deteriorated and was started on CPAP. Over the following days he received a combination of CPAP and nasal high flow oxygen, a course of dexamethasone and a cycle of remdesivir.

Nine days following admission he started to complain of chest pain and had palpable surgical emphysema. A chest radiograph revealed no obvious pneumothorax so he underwent a CT examination. This demonstrated extensive surgical emphysema in the neck and thorax with extensive pneumomediastinum and a very small right sided pneumothorax (Fig. 1). Evidence of severe COVID-19 pneumonitis was seen in the lungs. A decision was made to stop CPAP and administer high flow oxygen. The pneumothorax and pneumomediastinum were managed 
conservatively. The patient was gradually weaned off oxygen and underwent a period of in-patient rehabilitation. He was eventually discharged 20 days after admission.

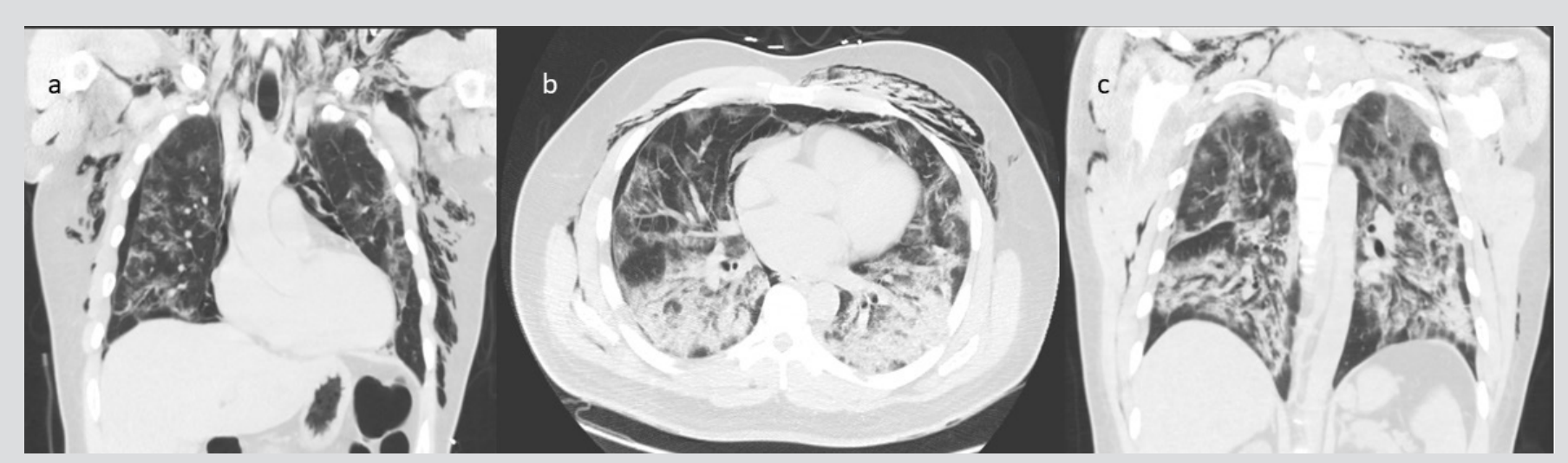

Figure 1. CT during first admission demonstrated extensive surgical emphysema in the neck and thorax with extensive pneumomediastinum and a very small right sided pneumothorax $(a, b)$. Evidence of severe COVID-19 pneumonitis was also seen in the lungs $(b, c)$.

Three days later he re-presented to the emergency department with sudden onset left sided chest pain and shortness of breath. He had a cough productive of clear sputum and had had one episode of haemoptysis. He had an increased respiratory rate and a sinus tachycardia. On auscultation a few scattered crepitations were noted in the left lower lung zone. The initial differential diagnoses were considered to be a community acquired pneumonia, a pneumothorax, or a pulmonary embolus.

Bloods tests revealed a mildly elevated CRP, an elevated D-dimer and a slightly elevated white blood cell count. Repeat tests for COVID-19 were negative. A chest radiograph (Fig. 2) revealed a new, small left sided pneumothorax with a lucency projected over the heart concerning for a loculated component. The clinical team thought that the pneumothorax was unlikely to explain the patient's hypoxia. They proceeded to request a CT pulmonary angiogram (CTPA) to evaluate the possibility of a pulmonary embolus (PE).

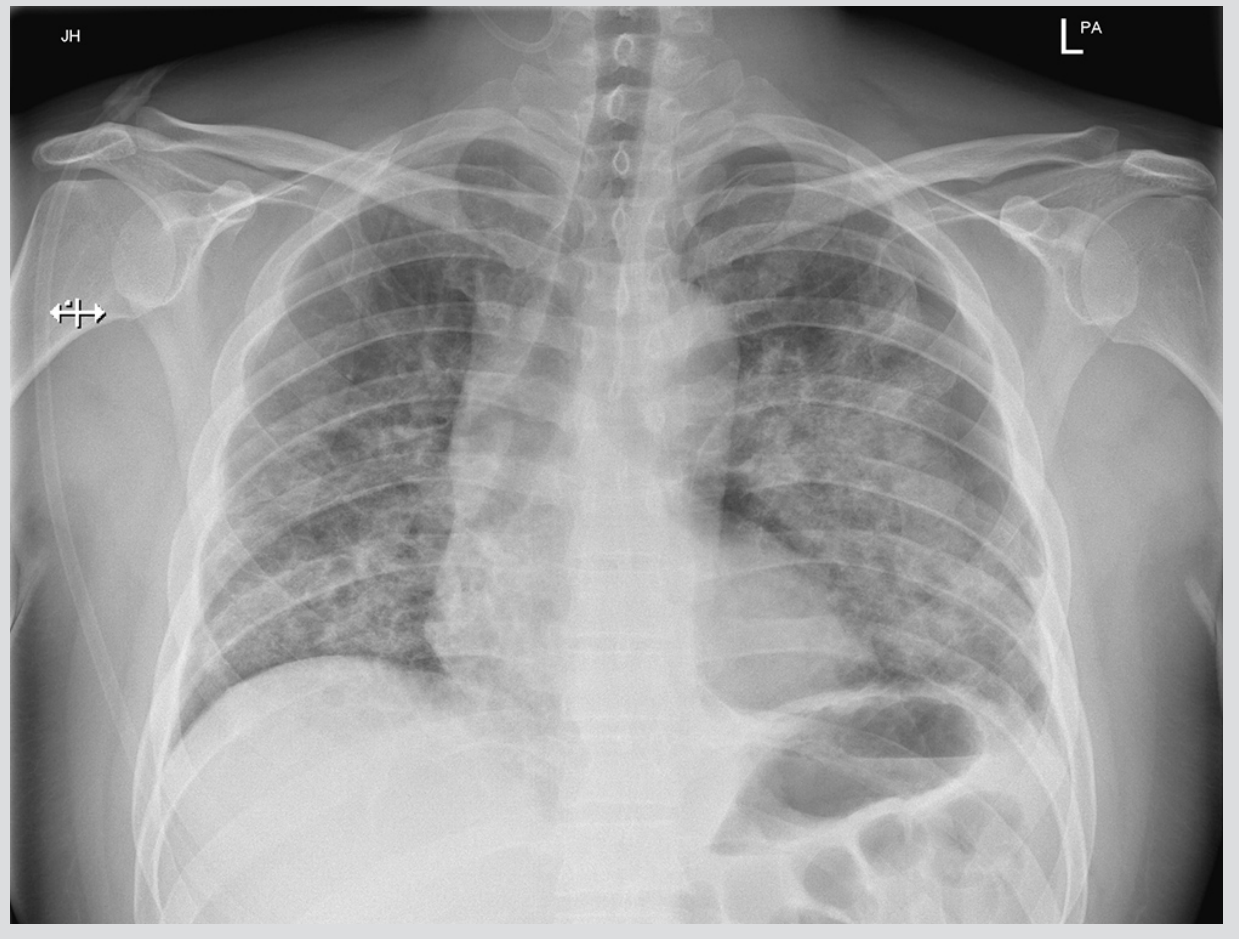

Figure 2. Initial chest radiograph on the second admission revealed a new, generally small left sided pneumothorax with a larger lucency projected over the heart concerning for a loculated component. 
There was no evidence of a PE on the CTPA but it demonstrated what was thought to be a new, moderate volume left sided pneumothorax with inferior loculation and some tethering of the lung (Fig. 3). There was associated left lower lobe atelectasis and a small volume of left pleural fluid. Two small new sub pleural cysts were demonstrated in the left lower lobe and lingula. There were also extensive bilateral residual changes in the lungs in keeping with the recent severe COVID-19 pneumonitis. The previously demonstrated pneumomediastinum and right sided pneumothorax had resolved.

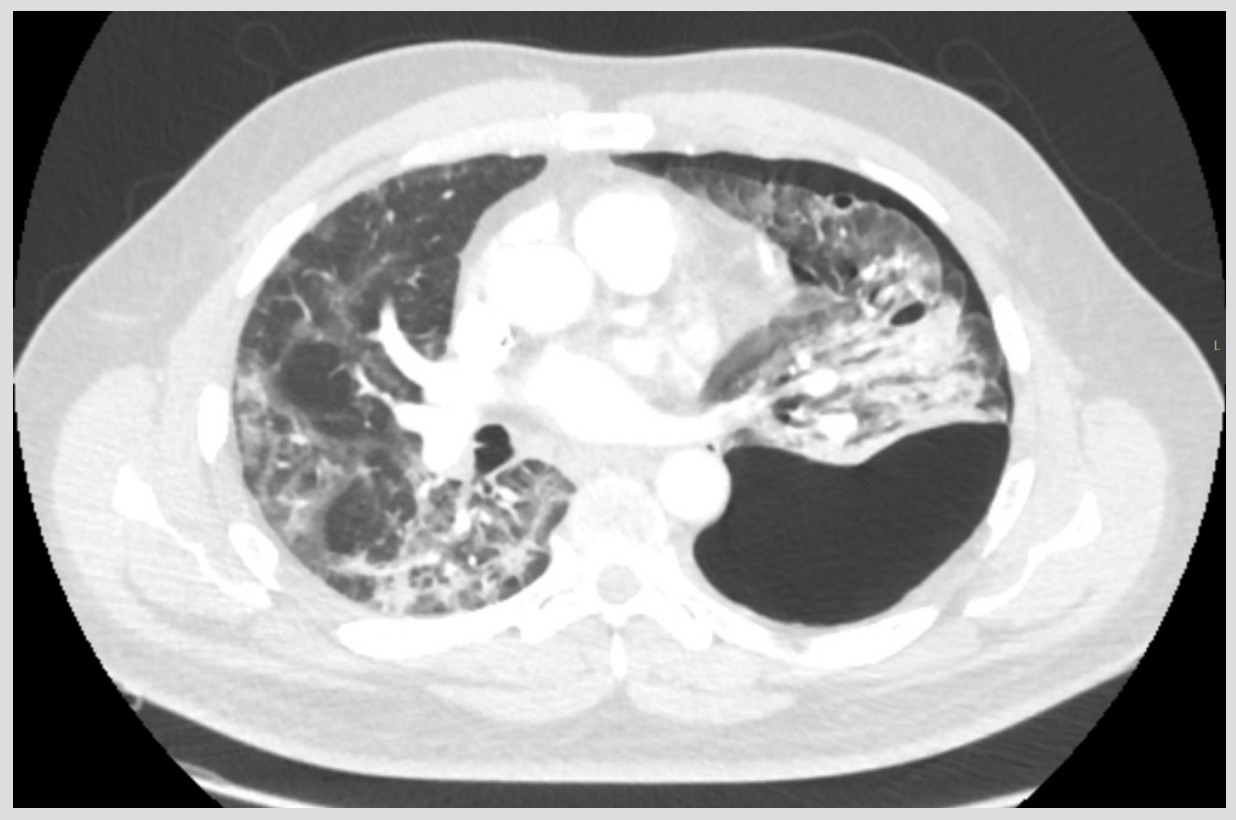

Figure 3. CTPA on the second admission revealed what was thought to be a new, moderate volume left sided pneumothorax with inferior loculation and some tethering of the lung. Two small new sub pleural cysts were demonstrated in the left lower lobe and lingula. There were also extensive bilateral residual changes in the lungs in keeping with the recent severe COVID-19 pneumonitis.

Ongoing clinical concerns led to a plan to drain the apparent left sided pneumothorax under CT guidance. However, when the patient was turned into the prone position in order to undergo this procedure, the presumed pneumothorax was revealed to be a large new left lower lobe pneumatocele with only a very small left sided pneumothorax (Fig. 4). As a result a chest drain was not inserted and conservative management with oxygen therapy and analgesia continued. The case was discussed with our local cardio-thoracic surgical team who advised against any surgical intervention until the COVID-19 related lung changes improved.
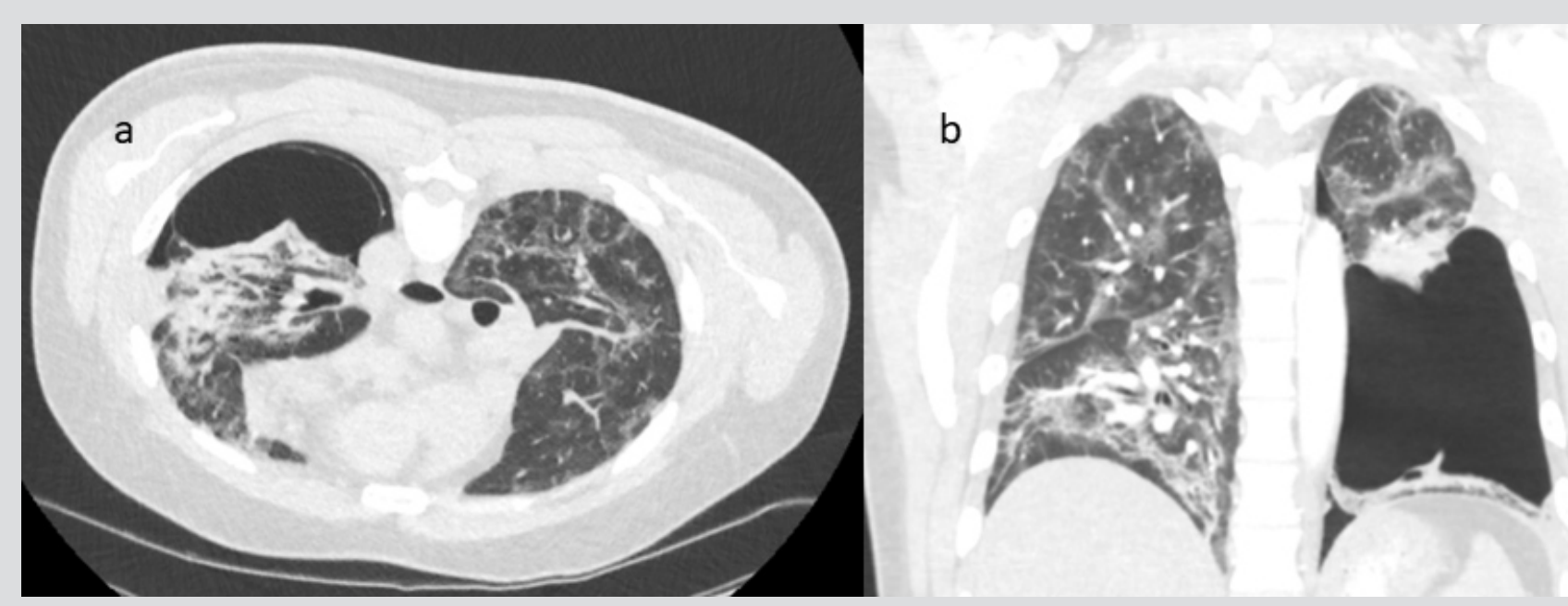

Figure 4. CT examination performed when the patient was turned into the prone position in order to undergo chest drain insertion. The presumed pneumothorax was revealed to be a large new left lower lobe pneumatocele with only a very small left sided pneumothorax $(a, b)$. 
The patient continued to improve clinically and was discharged home after 11 days with ambulatory oxygen. He was reviewed in the respiratory medicine clinic three weeks later. A repeat chest radiograph (Fig. 5a) demonstrated a decrease in the size of the thin walled pneumatocele. It also demonstrated resolution of the left sided pneumothorax and marked improvement of the COVID-19 pneumonitis related parenchymal changes. The patient's overall condition had improved. His home oxygen requirement had gradually decreased and his exercise tolerance was slowly improving. A plan was made to review him again in two months by which time he had further improved and no longer required ambulatory oxygen. A chest radiograph (Fig. 5b) demonstrated apparent resolution of the pneumatocele.

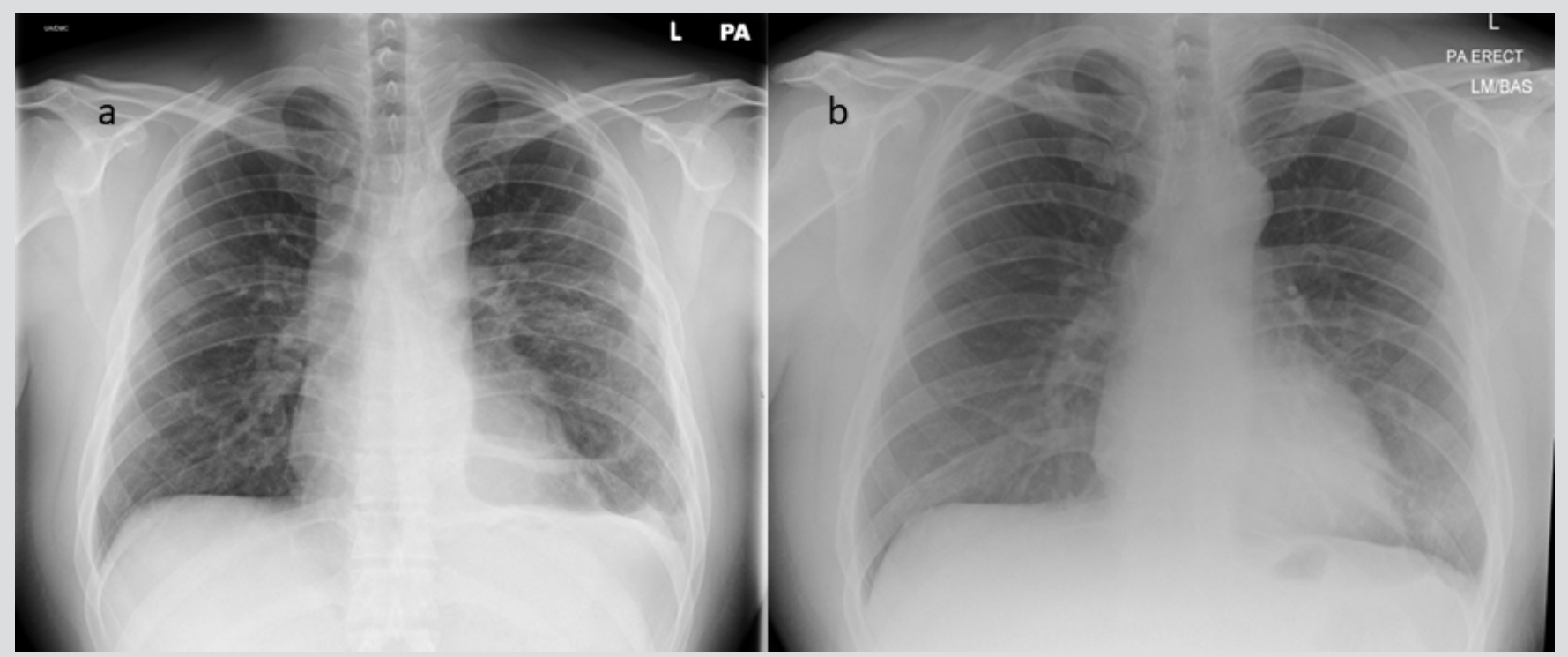

Figure 5. A repeat chest radiograph three weeks following final discharge (a) demonstrated an apparent decrease in the size of the thin walled pneumatocele with a small air-fluid level. It also demonstrated resolution of the left sided pneumothorax and marked improvement of the COVID-19 pneumonitis related parenchymal changes. Another chest radiograph (b) was performed after two months. This demonstrated apparent resolution of the pneumatocele.

\section{DISCUSSION}

This case demonstrates several potential complications related to COVID-19 pneumonitis. While pneumomediastinum and pneumothorax are fairly widely reported complications either resulting from mechanical or positive pressure ventilation and/or directly related to COVID-19 pneumonitis, the development of pneumatoceles secondary to COVID-19 pneumonitis appears much less common, particularly during convalescence ${ }^{[1-5]}$.

During his initial admission this patient did receive nine days of intermittent CPAP. While there is a link between positive pressure ventilation and pneumothoraces, there is no proven link between CPAP and pneumatocele formation. No bullae were demonstrated on the CT performed during the initial admission, the patient was a non-smoker with no relevant family history of lung disease and no evidence of alpha-1-antitrypsin deficiency. Events and appearances therefore strongly suggest that this is a case of delayed COVID-19 pneumonitisrelated pneumatocele formation.

This case illustrates the importance of CT imaging of the chest when the initial chest radiograph suggests the presence of a loculated pneumothorax. It also illustrates the fact that scanning patients in different positions may be prudent to help exclude the possibility of pneumatocele formation when a loculated pneumothorax is suspected on the supine images. This may help to prevent inadvertent drainage of pneumatoceles which may lead to continuous air leaks.

In conclusion, pneumatocele formation should be considered in the differential diagnoses of patients presenting with new respiratory symptoms after completing therapy for COVID-19 pneumonitis. CT imaging of the chest is essential to further evaluate possible complicated pneumothoraces diagnosed with chest radiographs. Acquiring images with patients in different positions may be required to differentiate pneumatocele formation from loculated pneumothoraces. 


\section{REFERENCES}

1. Sato L, Kinoshita N, Nakamoto T, Ohmagari N. Haemoptysis and a newly formed lung bulla in a case of convalescent COVID-19 pneumonia. Intern Med 2021;60(5):803-80.

2. Hamad AM, El-Saka HA. Post COVID-19 large pneumatocele: clinical and pathological perspectives. Interact Cardiovasc Thorac Surg 2021 March 25:ivab072. Online ahead of print

3. Sugimoto H, Era Y, Sugimoto K. Pneumatocele after recovering from COVD-19. BMJ Case Rep 2021; 14(4):e242729.

4. Chen Y, Chen W, Zhou J, Sun C, Lei Yujie. Large pulmonary cavity in COVID-19 cured patient case report. Ann Palliat Med 2020 Jun 9:apm-20-452. doi: 10.21037/apm-20-452. Online ahead of print.

5. Odackal J, Milinic T, Amass T, Chan ED, Hua J, Krefft S. A 28-year-old man with chest pain, shortness of breath, and hemoptysis after recovery from coronavirus disease 2019 pneumonia. Chest 2021 Jan;159(1):e35-e38. 